\title{
The usefulness of an intra-oropharyngeal u-turn method using trans-nasal endoscopy for detecting superficial squamous cell carcinoma of the base of the tongue
}

\begin{abstract}
Background: Recently, an increase in the incidence of oropharyngeal squamous cell carcinoma has been noted in the United States. Squamous cell carcinoma of the base of the tongue is often diagnosed at an advanced stage, with a dismal prognosis. With advances in endoscopy, more cases of superficial cancer are now being found. However, observing the base of the tongue using trans-oral endoscopy is difficult.

Aim: Starting in April 2012, we introduced a new technique for observing the base of the
tongue using trans-nasal esophagogastroduodenoscopy-known as the intra-oropharyngeal
U turn method. Its efficacy is reported here.

Results: Eleven cases of superficial squamous cell carcinoma of the base of the tongue were detected using trans-nasal endoscopy between April 2012 and March 2016. Five lesions were flat type, four were elevated, and two were depressed. Regarding the tumor size, eight were T1, and three were T2. No lymph node metastasis was detected in any case. Seven were resected, and three were treated with chemo-radiotherapy, while one was followed because of esophageal cancer. For the seven resected specimens, the median tumor

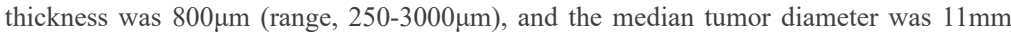
(range, 10-25mm). Histologically, 2 of these lesions were squamous cell carcinoma in situ, and five showed microinvasion of the subepithelial tissue. During a median followup period of 26months (range, 11-56months), 1 patient developed lymph node metastasis, which was treated by neck lymphadenectomy and chemo-radiotherapy. Three of the 11 patients died of another disease, and 8 were alive as of drafting this manuscript.
\end{abstract}

Conclusion: The intra-oropharyngeal U-turn method with trans-nasal endoscopy is useful for detecting superficial squamous cell carcinoma of the base of the tongue.

Keywords: Oropharyngeal squamous cell carcinoma, Subepithelial tissue, Chemoradiotherapy, Neck lymphadenectomy
Volume 8 Issue 2 - 2017

\author{
Kenro Kawada,' Tatsuyuki Kawano,' \\ Takuya Okada,' Kazuya Yamaguchi, I Yuudai \\ Kawamura,' Toshihiro Matsui,' Masafumi \\ Okuda,' Taichi Ogo, I Yuuichiro Kume,' \\ Takuya Okada,' Akihiro Hoshino,' Yutaka \\ Tokairin, ' Yasuaki Nakajima,' Ryuhei Okada, ${ }^{2}$ \\ Yusuke Kiyokawa, ${ }^{2}$ Fuminori Nomura, ${ }^{2}$ \\ Yosuke Ariizumi,2 Takashi Ito ${ }^{3}$ \\ 'Department of Gastrointestinal Surgery Tokyo Medical and \\ Dental University, Japan \\ ${ }^{2}$ Department of Head and Neck surgery Tokyo Medical and \\ Dental University, Japan \\ ${ }^{3}$ Department of Human Pathology Tokyo Medical and Dental \\ University, Japan
}

\section{Correspondence: Kenro Kawada Assistant professor Department of Gastrointestinal surgery Tokyo Medical and Dental University Tokyo, Japan, Email kawada.srg।@tmd.ac.jp}

Received: July 24, 2017| Published: August 01, 2017
Abbreviations: IEE, image-enhanced endoscopy; NBI, narrow band imaging; EGD, esophagogastroduodenoscopy; LCI, linked color imaging; FICE, flexible spectral imaging color enhancement

\section{Introduction}

Squamous cell carcinoma of the base of the tongue often is diagnosed at an advanced stage, typically in malnourished patients with a history of smoking and alcoholism. In the United States, an increase in the incidence of oropharyngeal squamous cell carcinoma has been noted. ${ }^{1}$ This increase has been predominantly for cancers involving the tonsil ( $4 \%$ per year) and the base of the tongue ( $2 \%$ per year). ${ }^{2}$ The early detection of cancer offers the best prognosis.

Recently, image-enhanced endoscopy (IEE) has been carried out to diagnose gastrointestinal tumors. Lugol chromoendoscopy can be used to detect superficial esophageal cancer, but it cannot be used for head and neck squamous cell carcinoma screening because of the risk of aspiration. The narrow band imaging (NBI) system is an imageenhanced technology that uses NBI filters. ${ }^{3}$ Muto et al., ${ }^{4-5}$ reported that NBI combined with magnifying esophagogastroduodenoscopy (EGD) was usuful for identifying early-stage or superficial carcinoma within the oropharyngeal and hypopharyngeal regions. A rhinolaryngoscope equipped with an NBI system is also available for examining these lesions. ${ }^{6}$

There are, however, some areas that are difficult to observe on trans-oral EGD. In particular, only a tangential view of the base of the tongue is obtained with trans-oral EGD. We previously reported the usefulness of the intra-oropharyngeal U-turn method using transnasal EGD which we used to diagnose superficial squamous cell carcinoma of the base of the tongue. ${ }^{7-8}$ The LASERIO system (Fuji Film, Co., Tokyo, Japan) is the first gastrointestinal endoscopy system to use laser light as illumination. ${ }^{9-10}$ It is equipped with a narrow-band light observation function that takes advantage of the characteristics of laser light, as well as a white light observation function. The linked color imaging (LCI) function is a new image enhancing technology available for the LASERIO system that makes slight differences in mucosal color tones easier to recognize. ${ }^{11}$

\section{Aim}

In this report, we present 11 cases of superficial squamous cell carcinoma of the base of tongue, that were detected by trans-nasal EGD (EG-580NW, EG-L580NW, Fuji Film, Co.) using the intraoropharyngeal U-turn method. 


\section{Materials and methods}

\section{Patients}

According to the 'field cancerization' concept, head and neck cancer, especially pharyngeal cancer, frequently coexist with esophageal cancer. ${ }^{12}$ We have routinely performed the intra-oropharyngeal U-turn method using trans-nasal EGD for patients with esophageal cancer or head and neck cancer since April 2012.

\section{Preparation}

Trans-nasal EGD can be performed comfortably because of its attenuation of the gag reflex. The trans-nasal endoscopic examination is performed without sedation in Japan. Prior to commencement of the procedure, each nasal cavity was sprayed with $0.05 \%$ naphazoline nitrate to induce vasoconstriction, followed by premedication with $100 \mathrm{mg}$ of dimethylpolysiloxane and $10000 \mathrm{U}$ of pronase, with sodium bicarbonate to remove mucus and foam in the stomach. Thesis was started by spraying a solution of $4 \%$ lidocaine into the nostril for three minutes, after which a swab covered with $8 \%$ lidocaine spray was inserted into the deeper nasal cavity for two minutes. The patient was then placed in the lateral decubitus position to receive endoscopy. Antispasmodics such as scopolamine were not used for premedication.

\section{Equipment}

There are some disadvantages to this procedure compared with conventional endoscopy, such as a decrease in the brightness, image resolution and suction ability, so the diagnostic abilities of this system have not been sufficiently evaluated. However, the procedure has recently been making tremendous progress, and an new type of endoscope (model EG-580NW, FUJI Film, Tokyo, Japan) was manufactured beginning in October of 2011. The outer diameter of the endoscope is $5.9 \mathrm{~mm}$. This endoscope is a trans-nasal model that provides high-quality endoscopic images with a wide field view (of $140^{\circ}$ ), and is equipped with the flexible spectral imaging color enhancement (FICE) system, which enhances images by extracting spectral images at the desired wavelengths via the application of signal processing to the white light generally used by endoscopes. ${ }^{13-14}$ However, given the limited ability of conventional white light illumination to achieve high-cotrast imaging of microvessels in the mucosal surface layer, a more effective system was needed. A newgeneration endoscopy system called LASERIO, was introduced by Fuji film in September 2012. It is equipped with not only a white light observation but also a new narrow-band light observation function that takes advantage of the characteristics of laser light. Blue laser imaging observation (BLI) using the LASERIO system has successfully achieved a bright and clear image even at a distance. ${ }^{9-10}$ In 2014, the EG-L580NW was released by Fuji film Corporation. This new endoscope is a thin endoscope that can pass through the patient's nose, equipped with LASERIO system.

The LASERIO system equipped with four visualization modes: BLI mode, BLI-bright mode, LCI mode (mentioned above), and white light mode. To change the mode, the endoscopist simply selects the desired mode on the operating portion of the endoscope with a button.

\section{Screenig procedure}

Our screening procedure is as follows: First, the patient is asked to bow their head deeply in the lateral decubitus position. We then place a hand on the back of the patient's head and push it forward. The patient is then asked to lift their chin as far as possible (lateral sniffing position). In order to avoid overlooking cancers in the floor of the mouth, soft palate and uvula, we first observe the oral cavity. We then insert the endoscope without a mouthpiece and subsequently observe the upper, lateral and posterior wall of the oropharynx while the patient sticks their tongue out. After observing the buccal cavity, further oropharyngeal observation is carried out with a retroflexed endoscope inserted via the nose. When the tip of the endoscope reaches the area caudal to the uvula, the patient opens their mouth wide and sticks their tongue out as far as possible while making a vocal sound similar to "a long "I". The endoscopist then forces the endoscope to make a U-turn (intra-oropharyngeal U-turn method) and observes the oropharynx, in particular the base of the tongue. The procedure for performing the intra-oropharyngeal U-turn methods illustrated in Figure 1.

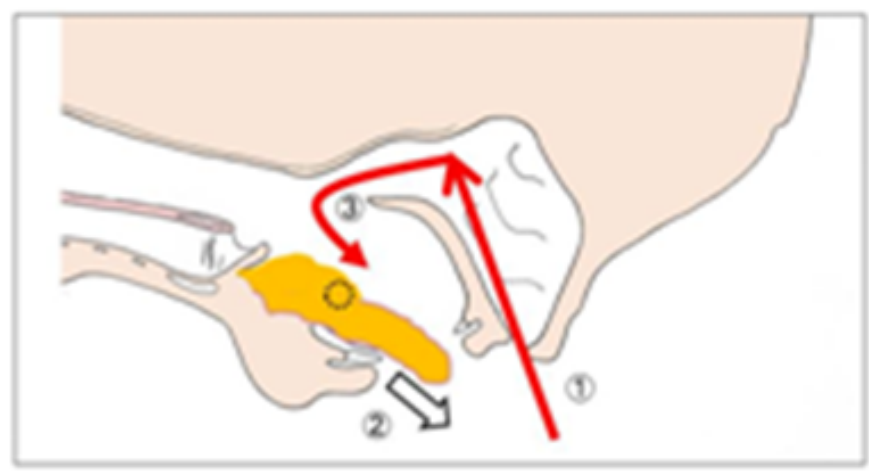

Figure I Schematic illustration of the intra-oropharygeal U-turn method.

Suction, irrigation, and insufflation are all possible with this transnasal flexible endoscope, which facilitates the clinical assessment of this particular area. The tip of the endoscope can make a U-turn up to an angle of $210^{\circ}$. We previously reported the usefulness of the intraoropharyngeal $\mathrm{U}$ turn method. ${ }^{7-8} \mathrm{We}$ were able to observe all areas of the tongue from the base (Figure 2A) to the apex (Figure 2B) and a frontal view of the papillae vallatae was obtained in all patients. The squamous cell carcinoma of the base of the tongue detected by EGL580NW is shown in Figures 3A (white light imaging) and 3B (LCI). After completing the intra-oropharyngeal U-turn method, the tip of the endoscope is inserted gently between the epiglottis and the tongue to observe the vallecula and the tonsil side of the epiglottis.

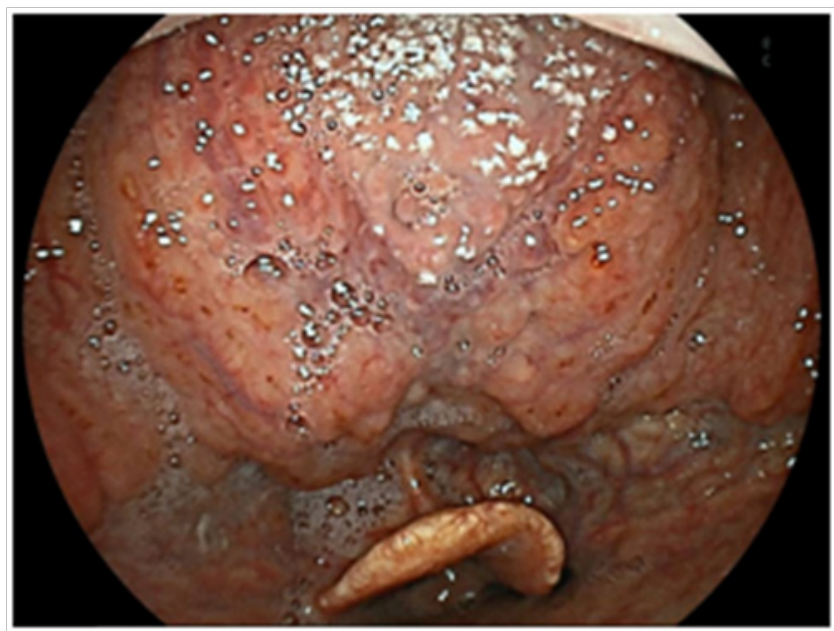

Figure 2A An endoscopic image obtained using the intra-oropharyngeal $U$ turn method. (A) The image of the base of the tongue. 


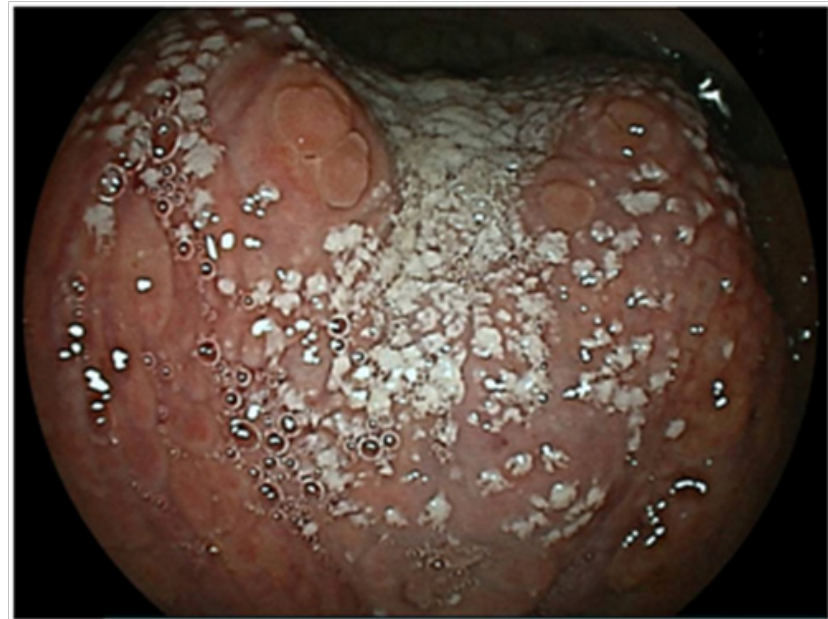

Figure 2B The image of the apex of tongue.

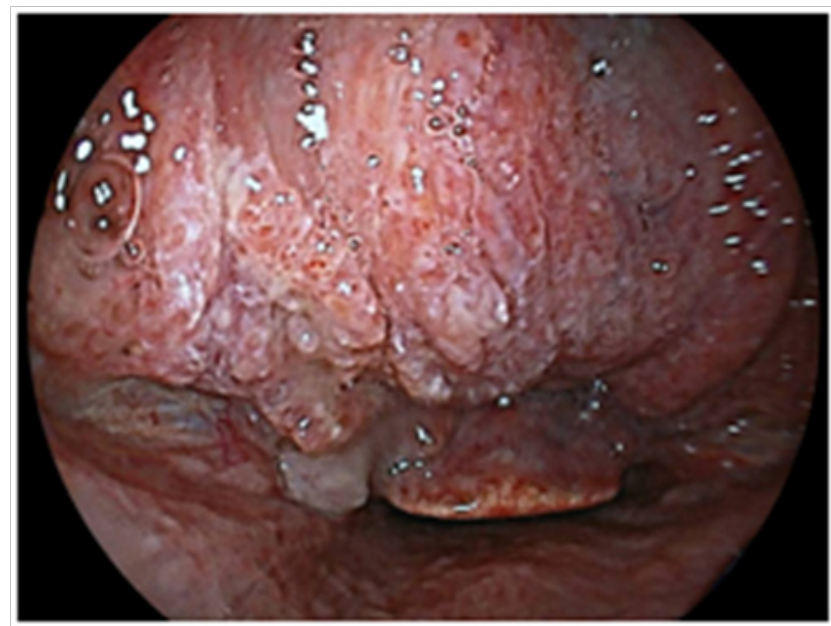

Figure 3A Superficial squamous cell carcinoma of the base of the tongue with white-light imaging.

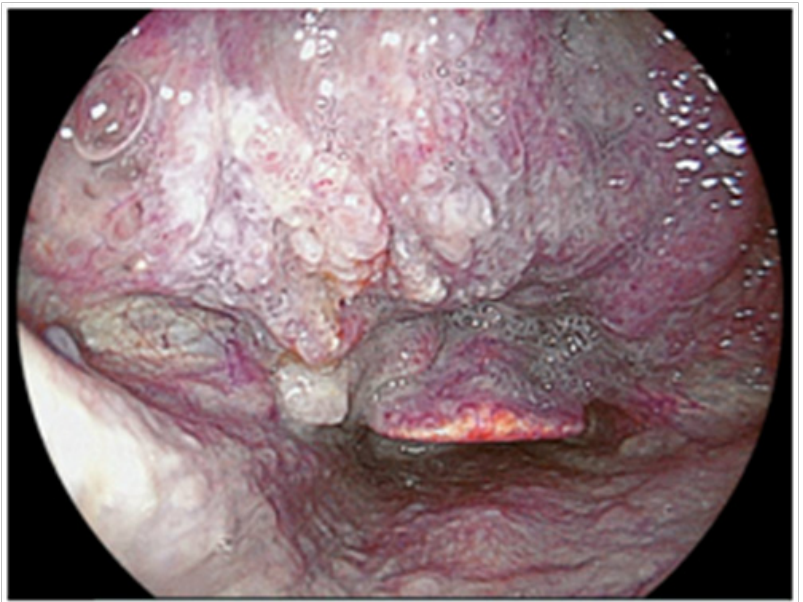

Figure 3B An image obtained with linked color imaging which enhances slight color differences in the red region of the mucosa. We can obtain bright images from a distance.

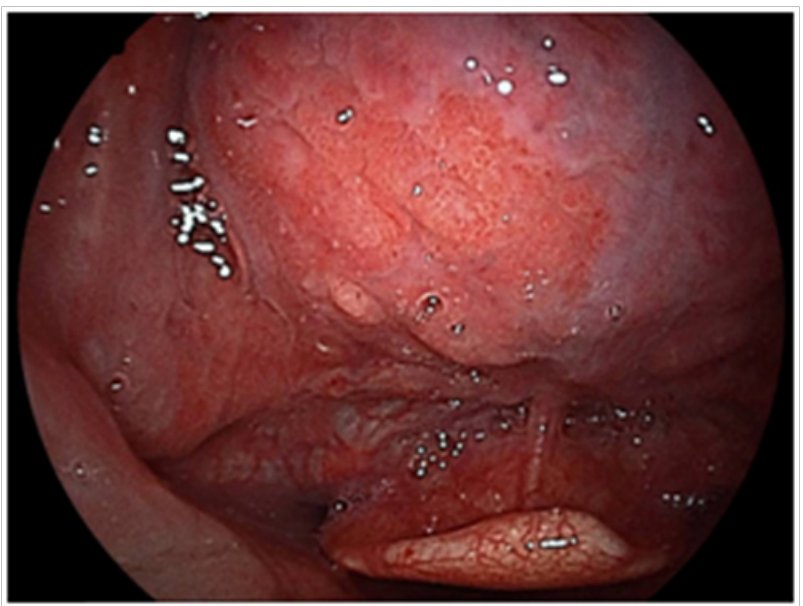

Figure 4A Flat type

Macroscopic types of superficial squamous cell carcinoma of the base of the tongue.

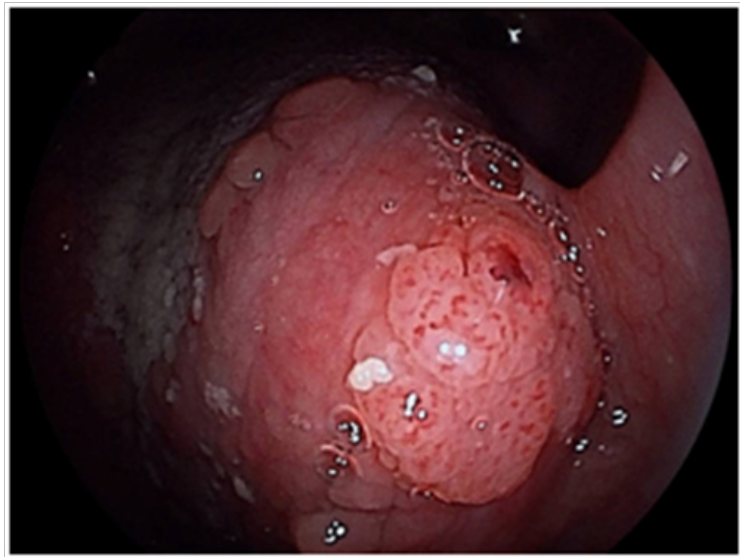

Figure 4B Elevated type.

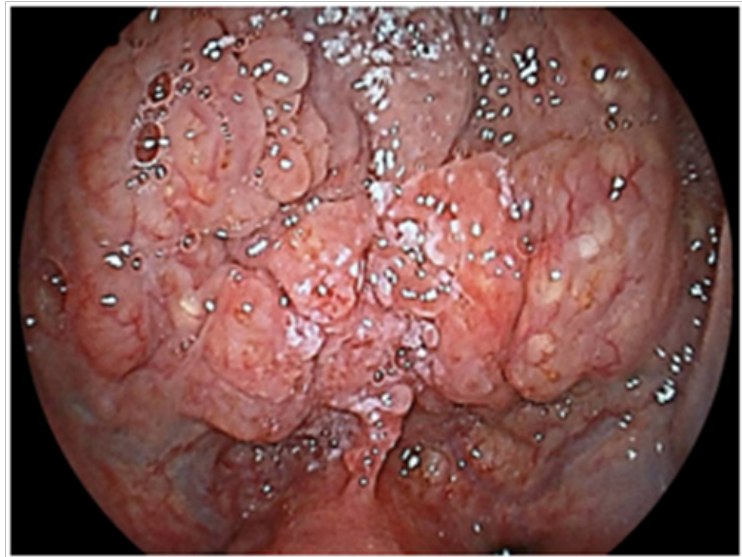

Figure 4C Depressed type.

We detected 11 cases of superficial squamous cell carcinoma of the base of the tongue between April 2012 and March 2016. Al the patients were complicated with current $(n=3)$ or previous $(n=8)$ esophageal squamous cell carcinoma. The patient characteristics are shown in Table 1. The mean age of the patients was 63.7years (range, 
49-73years), and all patients were male. Four cases were diagnosed using trans-nasal endoscopy with the FICE system, seven cases were diagnosed using trans-nasal endoscopy with the LASERIO system. The average time to complete a thorough examination for one patient was approximately from 1 to 2 minutes, regardless of the presence of abnormal findings. Biopsy specimen were obtained in all patients, and diagnosed as squamous cell carcinoma pathologically. Table 2 shows the treatment results. Five lesions were flat type (Figure 4A), four were elevated (Figure 4B), and two were depressed (Figure 4C). Regarding the tumor size, eight were T1, three were T2. No lymph node metastasis was detected in any case. Six cases were treated by endoscopic laryngo-pharygeal surgery, ${ }^{15}$ and 3 cases were treated by chemo-radiotherapy. One case was followed because of esophageal cancer. One case was treated by trans-oral robotic surgery at another hospital. For the seven resected specimens, the median tumor thickness was $800 \mu \mathrm{m}$ (range, $250-3000 \mu \mathrm{m}$ ), and the median tumor diameter was $11 \mathrm{~mm}$ (range, $10-25 \mathrm{~mm}$ ). Histologically, two of these lesions were squamous cell carcinoma in situ, five showed microinvasion of the subepithelial tissue. During a median follow-up period of 26 months (range,11-56months), 1 patient, which patient's tumor thickness was $3000 \mu \mathrm{m}$, developed lymph node metastasis, which was treated by neck lymphadenectomy and chemo-radiotherapy. Three of the 11 patients cases were died of another disease, and 8 were alive as drafting this manuscript.

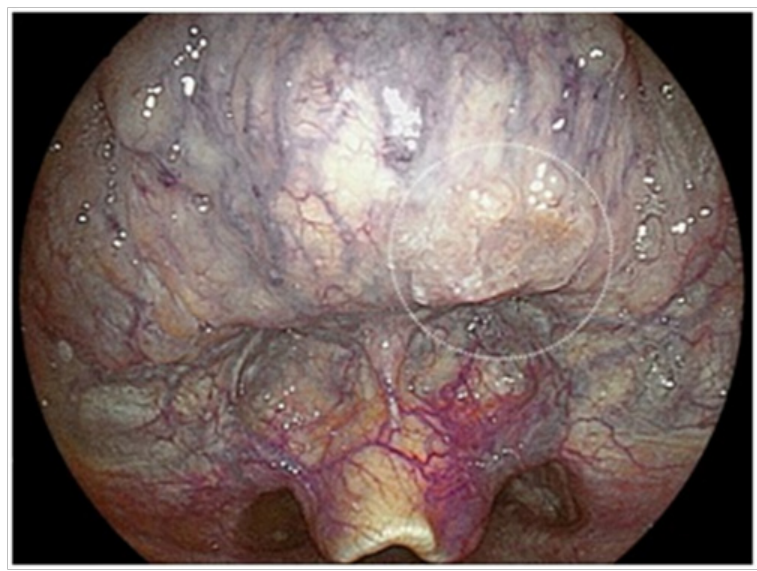

Figure 5A LCl mode at a distance.

Superficial squamous cell carcinoma detected by trans-nasal endoscopy using intra-oropharyngeal U-turn method.

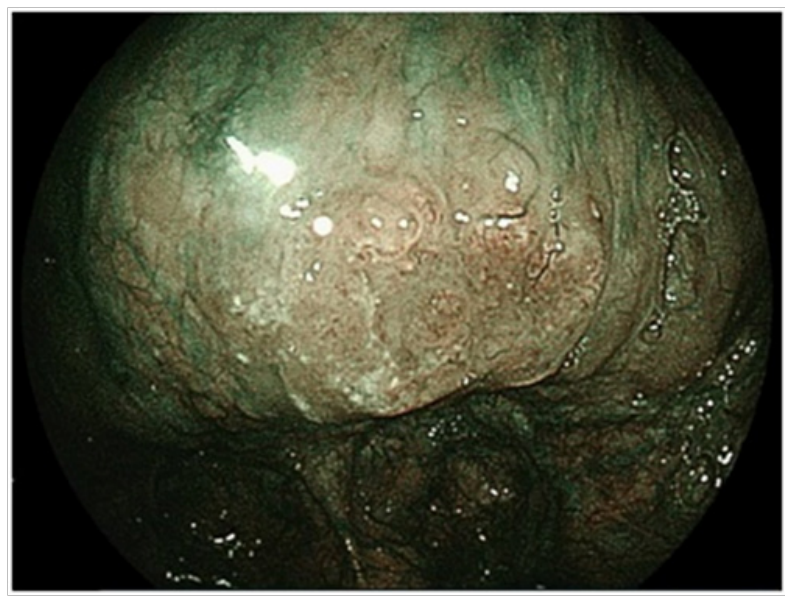

Figure 5B BLI mode close-up.

\section{Case presentation}

A 73-year-old male who had undergone esophagectomy for esophageal cancer 3 months previously was admitted to our hospital with primary hypopharyngeal cancer. Before treatment, upper gastrointestinal screening was performed by trans-nasal endoscopy (model EG-L580NW, Fuji film, Co.). Using the intra-oropharyngeal U-turn method, an elevated lesion located at base of the tongue was easily detected in LCI mode (Figure 5A). We were able to obtain a clear image of the tumor without magnification at a close view.

In BLI mode, irregular vessels around the tumor were clearly observed (Figure 5B). We were able to obtain a biopsy specimen without sedation, and confirmed the lesion to be squamous cell carcinoma. This patient had two other primary hypopharyngeal cancers, so he was treated by chemo-radiotherapy. This screening method is useful for a precise inspection before treatments in patients already known to have lesions.

Table I Characteristics of patients with base of the tongue carcinoma detected by trans-nasal EGD

\begin{tabular}{ll}
\hline \multicolumn{2}{l}{ Clinical Characteristics of Patients } \\
\hline Age, Years \\
\hline Mean & 63.7 \\
\hline Range & $49-73$ \\
\hline Sex & \\
\hline Mean & II \\
\hline Female & 0 \\
\hline Esophageal Cancer \\
\hline Present & $\mathrm{II}$ \\
\hline Synchronous & 3 \\
\hline Prior & 8 \\
\hline
\end{tabular}

Table 2 Treatment Results

$\begin{array}{llc}\text { Macroscopic Type } & \text { Flat } & 5 \\ & \text { Elevated } & 4 \\ & \text { Depressed } & 2 \\ \text { Tumor Size } & \text { TI } & 8 \\ & \text { T2 } & 3 \\ \text { Median Diameter of Tumor in } & \text { IImm (Range I0 25mm) } \\ \text { Resected Cases } & & \\ \text { Depth of Tumor } & \text { EP } & 2 \\ & \text { Subepithelial } & 5 \\ \text { Tumor Thickness (Median) } & 800 \mu m \text { Range }(250-3000)\end{array}$

\section{Discussion}

Multiple squamous cell carcinomas often arise in the upper aerodigestive tract. Patients with esophageal cancer often have multiple cancers, with squamous cell carcinoma of the head and neck occurring most often. ${ }^{16-18}$

The NBI system (Olympus Medical System Co., Tokyo, Japan) has been already proven to be a useful diagnostic tool for identifying superficial squamous cell carcinoma of the head and neck. ${ }^{4-6,19-21}$ Trans-nasal endoscopy can usually be performed comfortably due to attenuation of the gag reflex; ${ }^{22}$ indeed in Japan, trans-nasal endoscopy is a very common procedure and can be performed without sedation. It has also been reported that trans-nasal endoscopy is less invasive than other techniques with respect to the cardiopulmonary function. ${ }^{23-24}$ 
Furthermore, there are some disadvantages to using the trans-nasa endoscopy compared to conventional endoscopy, such as reductions in the image brightness, and resolution and a decreased suction ability, and its diagnostic abilities have not been evaluated. However recent advances with this procedure have been made, and a new endoscope (model EG-580NW, Fuji Film, Co.) were manufactured beginning in October 2011. This endoscope is a trans-nasal model that provides high-quality endoscopic images with a wide field view of $\left(140^{\circ}\right)$ and is equipped with the flexible spectreal imaging color enhancement (FICE) system, which is based on a spectral estimation technique by which the narrow bandwidth is obtained from white light images without optical filters. ${ }^{13-14}$ Unfortunately, the FICE system cannot image distinctive microvessels on the tumor surface as sharply as NBI.

A clear visualization of the microvasculature and microstructure of the tumor surface is ideal. BLI may be able to compensate for FICE because it uses narrow-band laser light combined with white light. Indeed BLI endoscopy has been shown to produce a new type of bright, high-resolution images of the digestive tract mucosa. ${ }^{9-10}$

In the present study, we used a new trans-nasal endoscopy device the EG-L580NW, (Fuji Film, Co.) with a LESERIO system (video processor: VP-4450; Fuji Film, Co.) including a light source (LL4450; Fuji Film, Co.) with a modified endoscopic technique to observe head and neck cancers and obtained excellent results. This system is equipped with two lasers with different wavelengths, one for whitelight sources (wavelength: $450 \mathrm{~nm}$ ), and one for BLI (wavelength: $410 \mathrm{~nm})$.

The BLI observation mode is useful for acquiring mucosal surface information, including the patterns of the surface blood vessels and structures. The endoscope allows for detailed observations with a close-up view. LCI is a new image-enhanced technology Available for the LASERIO system that makes slight differences in mucosal color tones easier to recognize. ${ }^{11}$ The LCI mode allows for detailed observation from a distance.

We previously reported the first case of superficial squamous cell carcinoma of base of the tongue diagnosed using the intraoropharyngeal U-turn method in $2012 .{ }^{8}$ We performed this endoscopic screening for high-risk patients, such as those with esophageal cancer and head and neck cancer, heavy drinkers, and heavy smokers. This provided a much better view of the lesion than had been possible with trans-oral EGD alone. In the 4years since the first case report, we have diagnosed 11 cases of superficial squamous cell carcinoma for the base of the tongue associated with esophageal cancer. Eight were these stage were stage I(T1N0M0) $\square$ and three were Stage II(T2N0M0) $\square$ A histopathological $\square$ examination revealed two cases of squamous cell carcinoma in situ.

Trans-nasal endoscopy with the LASERIO system now enables the wider observation of the mouth and can be used to obtain adequate information for diagnosing early cancers without magnification. While the intra-oropharyngeal U-turn method using trans-nasal endoscopy has not yet caught on in Japan, this technique is very easy to perform, and is not very expensive. We therefore expect this method to become a standard procedure for observing the base of the tongue in high-risk patients in the near future.

\section{Conclusion}

The intra-oropharyngeal U-turn method with trans-nasal endoscopy is useful for detecting superficial squamous cell carcinoma of base of the tongue.

\section{Acknowledgemnts}

None.

\section{Conflicts of interest}

Authors declare no conflict of interests for this article.

\section{Funding}

None.

\section{References}

1. Chaturvedi AK, Engels EA, Anderson WF, et al. Incidence trends for human papillomavirus-related and unrelated oral squamous cell carcinomas in the United States. J Clin Oncol. 2008;26(4):612-619.

2. Shiboski $\mathrm{CH}$, Schmidt BL, Jordan RC. Tongue and tonsil carcinoma: increasing trends in the U.S. population ages 20-44 years. Cancer. 2005;103(9):1843-1849.

3. Gono K, Yamazaki K, Doguchi N $\square$ et al. Endoscopic observation of tissue by narrowband illumination. Optical Review. 2003;10(4):211-215.

4. Muto M, Nakane M, Katada C, , et al. Squamous cell carcinoma in situ at oropha.ryngeal and hypopharyngeal mucosal sites. Cancer. 2004;101(6):1375-1381.

5. Muto M, Horimatsu T, Ezoe Y, et al. Improving visualization techniques by narrow band imaging and magnification endoscopy. J Gastroenterol Hepatol. 2009;24(8):1333-1346.

6. Watanabe A, Tsujie H, Taniguchi M, et al. Laryngoscopic detection of pharyngeal carcinoma in situ with narrow band imaging. Laryngoscope. 2006;116(4):650-654.

7. Kawada K, Okada $\mathrm{T}$, Sugimoto $\mathrm{T}$, et al. Intra-oropharyngeal U-turn method with Trans-nasal Endoscopy. The Japan Bronchoesophagological Society. 2013;64(4):265-270.

8. Kawada K, Okada T, Sugimoto T,et al. Intraoropharyngeal U-turn method using transnasal esophagogastroduodenoscopy. Endoscopy. 2014;46(Suppl 1UCTN):E137-E138.

9. Yoshida N, Hisabe Y, Imada M, et al. The ability of a novel blue laser imaging system for the diagnosis of invasion depth of colorectal neoplasms. J Gastroenterol.2013;49(1):73-80.

10. Osawa H, Yamamoto H. Present and future status of spectral imaging color enhancement and blue laser imaging technology. Dig Endosc. 2014;26(suppl 1):105-115.

11. Dohi O, Yagi N Onozawa Y, et al. Linked color imaging improves endoscopic diagnosis of active Helicobacter pylori infection. Endoscopy International Open. 2016;4(7):E800-E805.

12. Slaughter DP, Southwick HW, Smejkal W. Field cancerization in oral stratified squamous epithelium; clinical implications of multicentric origin. Cancer. 1953;6(5):963-968.

13. Osawa H, Yamamoto N, Yamada M, et al. Diagnosis of endoscopic Barrett's esophagus by transnasal flexible spectral imaging color enhancement. J Gastroenterol. 2009;44(11):1125-1132.

14. Osawa H, Yamamoto H, Miura M, et al. Diagnosis of extent of early gastric cancer using flexible spectral imaging color enhancement. World J Gastrointest Endosc. 2012;4(8):356-361.

15. Sato Y, Omori T, Tagawa M. Treatment of superficial carcinoma in hypopharynx. Nihon Jibiinkouka Gakkai Kaiho. 2006;109(7):581-586.

16. Kumagai Y, Kawano T, Nakajima Y, et al. Multiple Primary cancers associated with esophageal carcinoma. Surg Today. 2001;31(10):872-876. 
17. Natsugoe S, Matsumoto M, Okumura H, et al. Multiple primary carcinomas with esophageal squamous cell cancer: Clinicopathologic Outcome. World Journal of Surgery. 2005;29(1):46-49.

18. Watanabe A, Hosokawa M, Taniguchi M, et al. Head and neck cancer associated with esophageal cancer Auris Nasu Larynx. 2007;34(2):207-211.

19. Watanabe A, Taniguchi M, Tsujie H, et al. The value of narrow band imaging endoscope for early head and neck cancers. Otolaryngol Head Neck Surg. 2008;138(4):446-451.

20. Ugumori T, Muto M, Hayashi R, et al. Prospective study of early detection of pharyngeal superficial carcinoma with the narrow band imaging laryngoscope. Head Neck. 2009;31(2):189-194.

21. Piazza C, Cocco D, De Benedetto L, et al. Narrow band imaging and high definition television in the assessment of laryngeal cancer $\square$ a prospective study on 279 patients. Eur Arch Otorhinolaryngol. 2010;267(3):409-414.
22. Tsuboi M, AraiM, Maruoka D, Matsumura T, et al. Utility of unsedated transnasal endoscopy for pharyngeal observation during esophagogastroduodenoscopy. A prospective study to assess cardiopulmonary function. Scand J Gastrorenterol. 2013;48(7):884-889.

23. Kawai T, Miyazaki I, Yagi K, et al. Comparison of the effects on cardiopulmonary function of ultrathin transnasal versus normal diameter transoral esophagogastroduodenoscopy in Japan. Hepatogastroenterology. 2007;54(75):770-774.

24. Yuki M, Amano Y, Komazawa Y, et al. Unsedated transnasal smallcaliber esohagogastroduodenoscopy in elderly and bedridden patients. World J Gastroenterol. 2009;15(44):5586-5591. 